\title{
Reflow Process for Contact Hole ArF Lithography
}

\author{
Bang-Ching Ho, D. C. Owe-Yang, Shang-Ho Lin, Harrison Chen, J. C. Shih, Chia-Sui Hsu, J. H. Chen \\ Taiwan Semiconductor Manufacturing Company,
}

No. 8, Li-Hsin Rd. 6, Science-Based Industrial Park,

Hsin-Chu, Taiwan 300-77, R.O.C.

Keywords: reflow process, contact hole, ArF lithography

\section{Introduction}

The 2002 update of the international Technology Roadmap for Semiconductors (ITRS) lists the ADI's CD of contact hole resist for ASICs at the 65-nm node as 100 $\mathrm{nm}$ [1]. The depth of focus (DOF) for small contacts with the current 193-nm ArF exposure tools is inadequate for a manufacture that includes dense through isolated pitches. According to Rayleigh's equation, the higher numerical aperture (NA) 193-nm ArF exposure tools are not expected to improve DOF margins. Therefore it is very important to study the capability of process based resolution enhancement techniques to print small contacts using current 193-nm ArF exposure tools. In this paper, we study the reflow process for ArF contact hole photoresist to increase process windows of small contacts.

Printing of small contacts for the $65-\mathrm{nm}$ device generations will be a difficult challenge with ArF lithography capabilities. For the $65-\mathrm{nm} \mathrm{ASIC}$ generation, the 2002 update of the ITRS specifies the ADI's CD of contact hole resist as $100 \mathrm{~nm}$ [1]. One of the main challenges with the current $\mathrm{ArF}$ lithography processes is the insufficient common DOF of through-all-pitch small contacts. The higher NA 193-nm ArF exposure tools are expected to increase the exposure latitude and improve the

\section{After Development}

\section{Photoresist}

Received April 1, 2004

Accepted June 1, 2004 resolution at dense pitches, but DOF would will further decrease according to the Rayleigh's equation for DOF.

Improvements in 193-nm processes and contact hole photoresist are very important. The use of advanced reticle technology, such as attenuated phase shifting masks (AttPSM) with $6 \%$ or high transmission helps in increasing the DOF of isolated contact holes. Unfortunately, the dense patterns do not get benefit from these masks. Alternative illumination modes such annular and quadrupole offer benefit for dense contact patterns, but no benefit for isolated hole process window. Early work with 248-nm contact resists has shown that resist reflow can increase process window for small contacts [2][3]. Recently, some papers investigate feasibility of 193-nm contact photoresist reflow process [4][5][6]. In this paper, we study the reflow process for $\mathrm{ArF}$ contact hole photoresist to increase process windows of small contacts.

\section{Experimental}

Resist, ShinEtsu's 193nm COC resist material, was coated on $193 \mathrm{~nm}$ organic BARC substrates to reduce substrate reflectivity or footing effects. Hitachi 9360 is used to top-down SEM metrology, and Hitachi 4770S, for cross-section pictures.

\section{After Reflow}
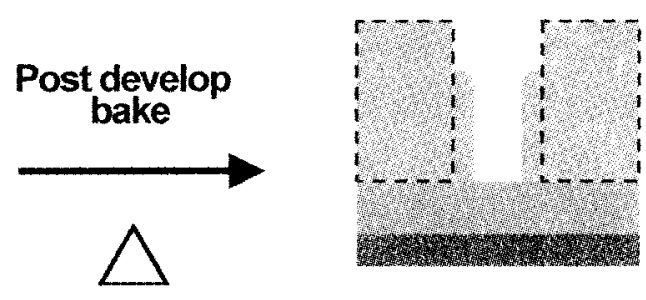

Figure 1 Reflow process scheme 
The scheming of the reflow process is shown in Figure 1. A high temperature baking step is used after the typical contact patterns have been developed in the resist. The post-development-bake-induced resist flow causes the contact features to shrink in size, leaving smaller final resist dimensions.

\section{Results and discussion}

There are three process-based resolution enhancement techniques to obtain smaller contacts, that is, Reflow process, RELACA (Resolution Enhancement Lithography Assisted by Chemical Shrink) and SAFIER (Shrink Assist Film for Enhancement Resolution) [6]. RELACS and SAFIER consist of water-soluble overcoat materials acting as shrink agent and additives to control pattem profile and shrink properties precisely. They will increase the processing cost and reduce throughput. They can also increase the higher defect risk. On the other hand, the Reflow process uses a post develop bake temperature higher than the glass transition temperature ( $\mathrm{Tg}$ ) of resist material. It has been a popular way of resolution enhancement technique due to the process simplicity and no loss in throughput.

\subsection{Optimization of process condition}

The resist used in this study, which is COC type ArF resist, has a lower $\mathrm{Tg}$ compared with that of the COMA or Acrylate platform. First, we study the effect of soft bake (SB) temperature \& post exposure bake (PEB) temperature, mask bias, and illumination mode for resist process window and profile. Based on these results, we can get larger process window and better resist profile.

\subsection{Reflow baking temperature optimization}

Shown in the Figure 2 is the shrinkage of $\mathrm{CD}$ for four different pitches, including $0.2 \mu \mathrm{m}, 0.22 \mu \mathrm{m}, 0.4 \mu \mathrm{m}$ and $2.0 \mu \mathrm{m}$, as a function of different bake temperature. Increasing the bake temperature gets more $\mathrm{CD}$ shrinkage and isolated contact holes shrink faster than the dense. At the same time, the iso-dense bias becomes large, and reflow bias for isolated contact holes become uncontrollable, as shown in Figure 3.

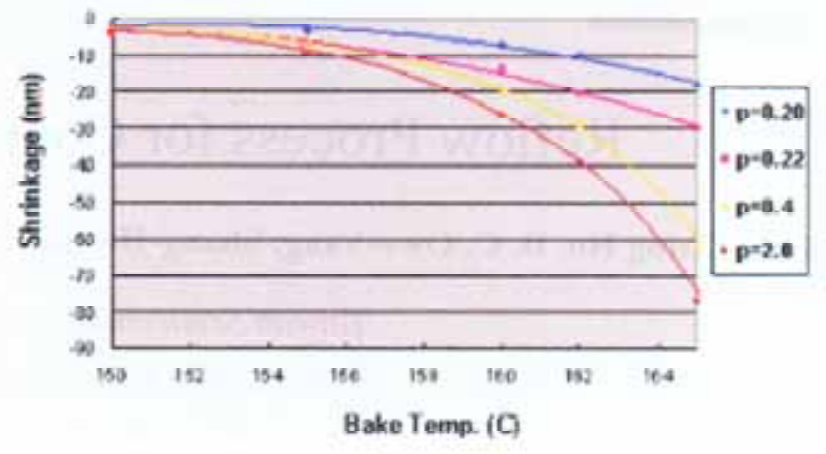

Figure 2. CD shrinkage vs, bake temperature

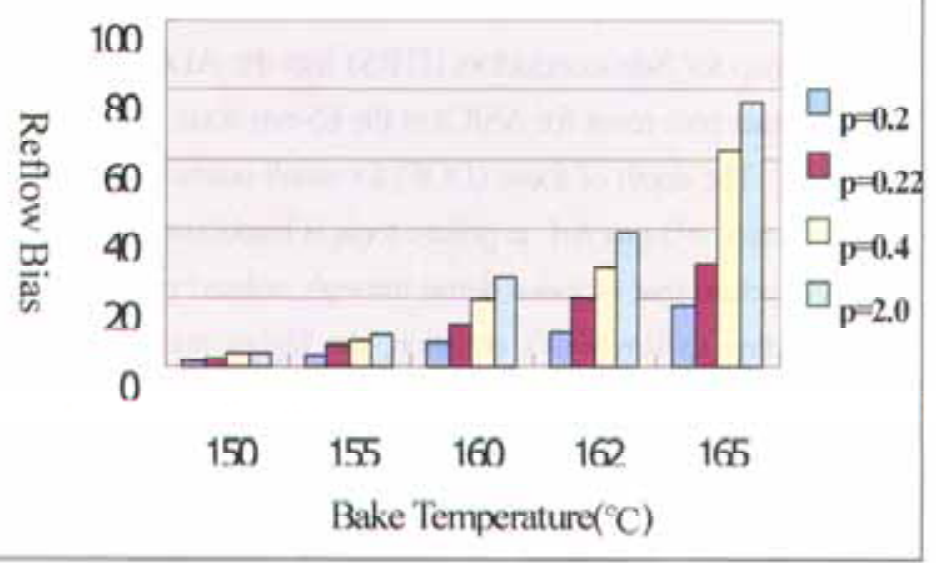

Figure 3. CD shrinkage vs, bake temperature

3.3 Process window after Reflow process

Process condition was optimized for 100 -nm contact holes through pitches. Figure 4 shows that before reflow process the through-all-pitch contact $C D$ is between

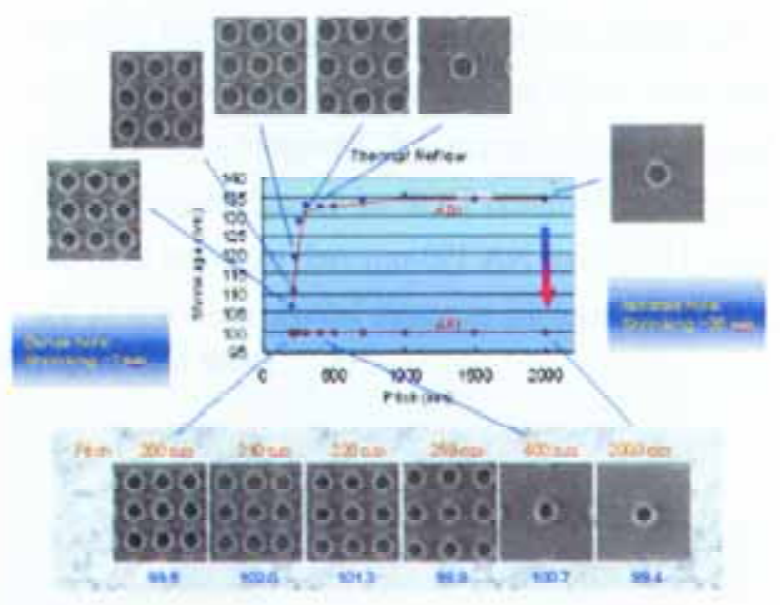

Figure 4. CD shrinkage vs, pitches. 
$107 \mathrm{~nm}$ and $135 \mathrm{~nm}$. After the reflow process the through-all-pitch contact $\mathrm{CD}$ meets the $100 \mathrm{~nm}$ target. The common process window ist $0.325 \mu \mathrm{m} @ 8 \% \mathrm{EL}$ for $100 \mathrm{~nm}$ small contact holes, as shown in Figure 5.
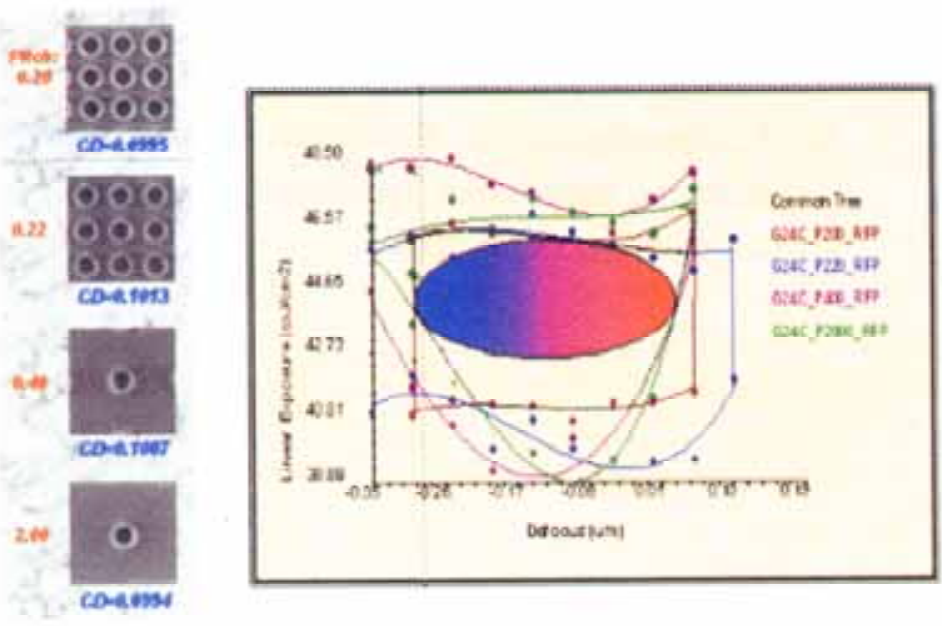

Figure 5. The common window of resist for $100 \mathrm{~nm}$ contact holes after reflow process

$3.5 \mathrm{CD}$ uniformity for $100 \mathrm{~nm}$ contact holes

Due to high $C D$ sensitivity to baking temperature, about $20 \mathrm{~nm}$ per degree for KrF resist, the CDU is around $12 \mathrm{~nm}$ and will be an issue for mass production. But in this study, the $\mathrm{CD}$ sensitivity of resist improves to below $5 \mathrm{~nm}$ per degree and its CDU can reach " 3 sigma $~ 2.5 \mathrm{~nm}$ " through pitches. It is much better than $\mathrm{KrF}$ resist. The results are shown in Figure 6.

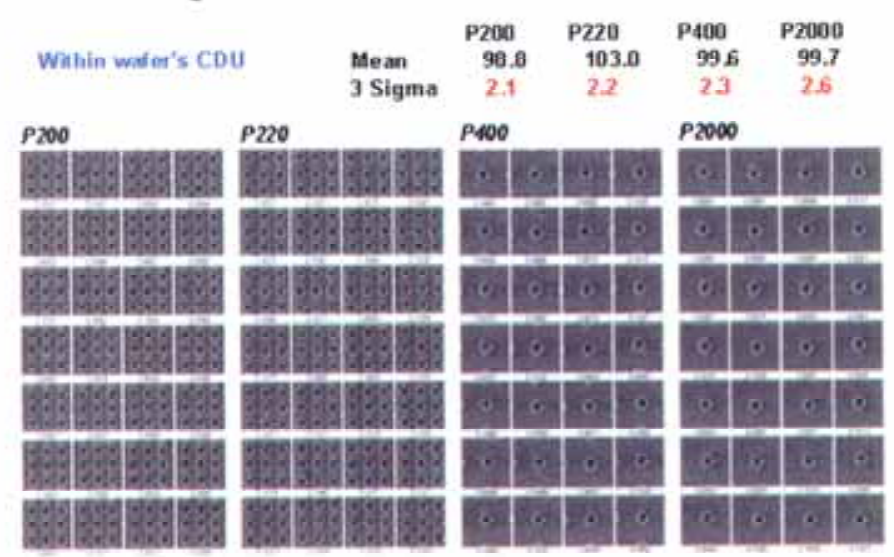

Figure 6.CDU for 100 -nm contact holes after reflow process

\subsection{Cross-section profiles}

Figure 7 shows that resist shrinkage and resist profile changes after the reflow process. The line-edge roughness of resist after the reflow process becomes smaller.

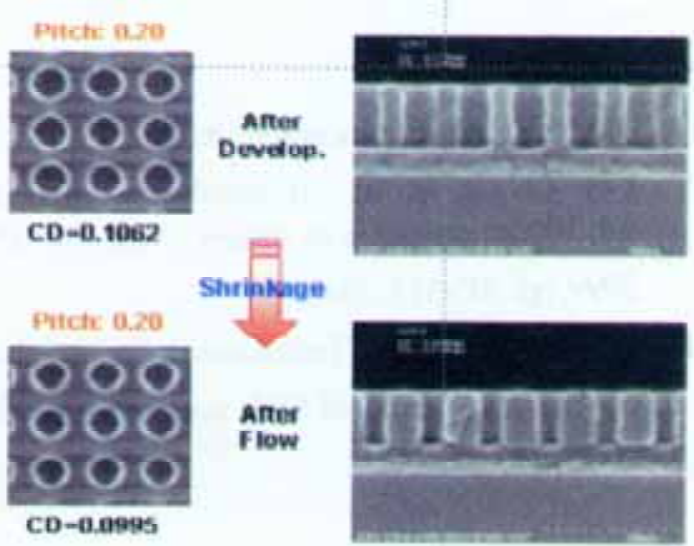

Figure 7. Cross-section pictures of resist before and after reflow process

\subsection{Proximity effects for SRAMs}

A SRAM cell demonstrates that our current OPC works with the Reflow processes. The top-view SEM pictures in Figure 8 show proximity issues. We still need to optimize the OPC recipe to reduce the proximity effect.

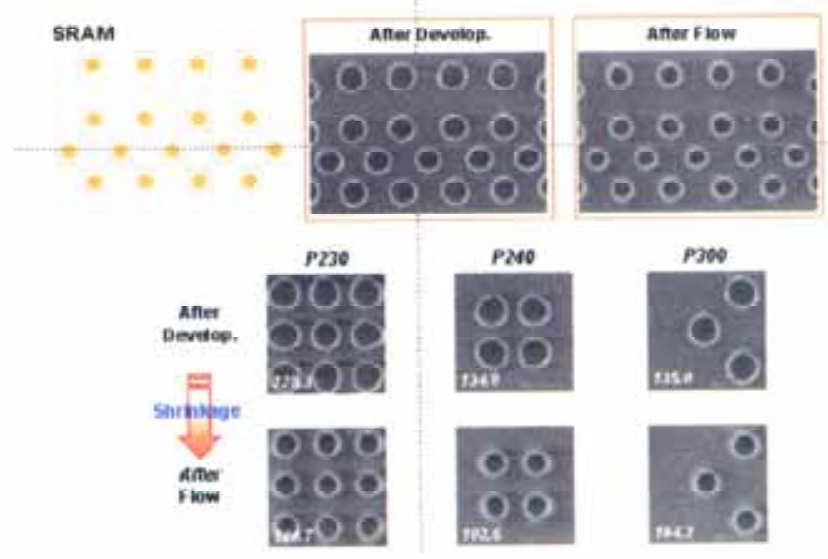

Figure 8. SRAM top-view SEM pictures before and after the Reflow process

\section{Conclusion}

We have developed significant improvement in process window using reflow resist in combination with the Reflow process. A through-all-pitch common DOF of $0.325 \mu \mathrm{m} @ 8 \% \mathrm{EL}$ and a cross-wafer CDU of $2.5 \mathrm{~nm}(3$ sigma) can be achieved. In the same time, we are printing smaller contact holes and pitches to tanget representative features for the 45 -nm node. 


\section{References}

1. 2002 TTRS ASIC Lithography Roadmap.

2. J.-H. Chung, et. al. "A novel resist material for sub-100nm contact hole pattern", Proc. of SPIE vol. 3999, pp. 305-312, 2000.

3. K. Aramaki, et. al."Techniques to print sub-0.2um contact holes", Proc. of SPIE vol. 3999, pp. 738-749, 2000.
4. K. Lucas, et. al. "193nm Contact Photoresist Reflow Feasibility Study" Prco. of SPIE vol. 4345, pp. 725-736, 2001.

5. P. Montgomery, et. al. "Resist Reflow for SPIE vol. 5039, pp.807-816, 2003.

6. S. Satyanarayana, et. al. "Evaluation of Process Based Resolution Enhancement Techniques", Proc. of SPIE vol. 5039, pp.257-268, 2003. 